\title{
Wind Information Uplink to Aircraft Performing Interval Management Operations
}

\author{
Nashat Ahmad*, Bryan Barmore ${ }^{\dagger}$, Kurt Swieringa ${ }^{\ddagger}$ \\ NASA Langley Research Center, Hampton, Virginia, 23681
}

\begin{abstract}
The accuracy of the wind information used to generate trajectories for aircraft performing Interval Management (IM) operations is critical to the success of an IM operation. There are two main forms of uncertainty in the wind information used by the Flight Deck Interval Management (FIM) equipment. The first is the accuracy of the forecast modeling done by the weather provider. The second is that only a small subset of the forecast data can be uplinked to the aircraft for use by the FIM equipment, resulting in loss of additional information. This study focuses on what subset of forecast data, such as the number and location of the points where the wind is sampled should be made available to uplink to the aircraft.
\end{abstract}

\section{Nomenclature}

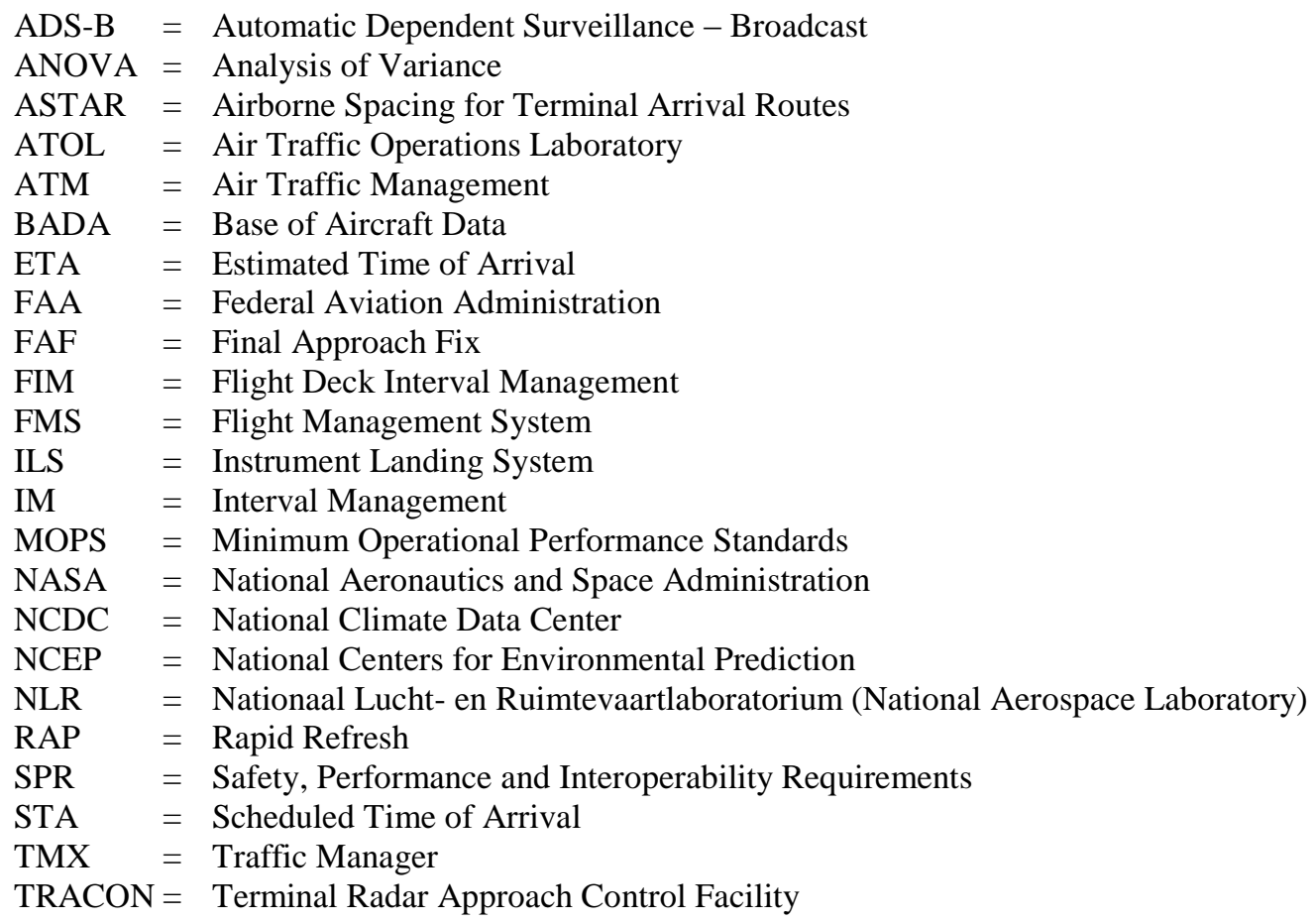

\section{Introduction}

Tnterval Management (IM) is an ADS-B-enabled suite of applications that use ground and flight deck capabilities 1 and procedures designed to support the relative spacing of aircraft (Barmore et al., 2004, Murdoch et al. 2009, Barmore 2009, Weitz et al. 2012). Relative spacing refers to managing the position of one aircraft to a time or distance relative to another aircraft, as opposed to a static reference point such as a point over the ground or clock time. This results in improved inter-aircraft spacing precision and is expected to allow aircraft to be spaced closer to the

\footnotetext{
* Research Aerospace Engineer, NASA, Hampton, Virginia. Senior Member, AIAA.

$\dagger$ Research Aerospace Engineer, NASA, Hampton, Virginia. Member, AIAA.

$\star$ Research Aerospace Engineer, NASA, Hampton, Virginia. Member, AIAA.
} 
applicable separation standard than current operations. Consequently, if the reduced spacing is used in scheduling, IM can reduce the time interval between the first and last aircraft in an overall arrival flow, resulting in increased throughput. Because IM relies on speed changes to achieve precise spacing, it can reduce costly, low-altitude, vectoring, which increases both efficiency and throughput in capacity-constrained airspace without negatively impacting controller workload and task complexity. This is expected to increase overall system efficiency.

The Flight Deck Interval Management (FIM) equipment provides speeds to the flight crew that will deliver them to the achieve-by point at the controller-specified time, i.e., assigned spacing goal, after the target aircraft crosses the achieve-by point. Since the IM and target aircraft may not be on the same arrival procedure, the FIM equipment predicts the estimated times of arrival (ETA) for both the IM and target aircraft to the achieve-by point. This involves generating an approximate four-dimensional trajectory for each aircraft. The accuracy of the wind data used to generate those trajectories is critical to the success of the IM operation.

There are two main forms of uncertainty in the wind information used by the FIM equipment. The first is the accuracy of the forecast modeling done by the weather provider. This is generally a global environmental prediction obtained from a weather model such as the Rapid Refresh (RAP) from the National Centers for Environmental Prediction (NCEP). The weather forecast data will have errors relative to the actual, or truth, winds that the aircraft will encounter. The second source of uncertainty is that only a small subset of the forecast data can be uplinked to the aircraft for use by the FIM equipment. This results in loss of additional information.

The Federal Aviation Administration (FAA) and RTCA are currently developing standards for the communication of wind and atmospheric data to the aircraft for use in NextGen operations. This paper examines the impact of various wind forecast sampling methods on IM performance metrics to inform the standards development.

\section{Experiment Design}

This study partially addressed the following two questions:

- What subset of the full wind forecast is needed by the FIM equipment to provide the desired IM performance?

- Is the accuracy of the forecast models sufficient to achieve the desired IM performance?

The first question was addressed by testing several different wind uplink options of the same forecast data and characterizing the effect on the IM performance metrics. To address the second question, an attempt was made to validate that the wind forecast accuracy values derived from other Air Traffic Management (ATM) applications are sufficient for IM operations. The following assumptions were made in this study as part of the testing and simulation:

- All uplink data comes from the same base forecast data.

- An IM aircraft will receive wind uplink data for their route of flight as well as for their target aircraft's route of flight. This information is assumed to be available at the time of the IM clearance.

- The FIM equipment will use all wind data provided to it to generate trajectories for the IM and target aircraft.

- Sensed winds on the IM aircraft will be used to update the internal wind model that is used for both the IM and target aircraft. No sensed data will be available from the target aircraft.

The Traffic Manager (TMX) (Bussink et al. 2005; Nuic 2003) was used as the simulation platform for this study. TMX is a fast-time modeling system developed by NLR and jointly maintained with NASA. A large set of simulations of different traffic scenarios can be performed using TMX by varying relevant initial conditions. TMX has flight management system (FMS) and ADS-B models and uses the Airborne Spacing for Terminal Arrival Routes (ASTAR) spacing algorithm developed by NASA (Abbott 2015). In this study, each traffic scenario consisted of a string of six aircraft. The first aircraft followed a selected target speed profile and the five remaining aircraft performed IM as a string of aircraft. In this section, the selected wind uplink options and the initial conditions used in various traffic scenario simulations are described in detail.

\section{A. Airspace Environment}

This simulation investigated IM operations at the Phoenix Sky Harbor airport, using the west flow configuration. A total of three arrivals were simulated:

- CORKR transition, MAIER5 arrival;

- GUP transition, EAGUL5 arrival; and

- SSO transition, KOOLY4 arrival.

All three routes terminated at Runway 26 using the ILS26 approach. The routes are shown in Figure 1 (Page 3). 


\section{B. Uplink Wind Options}

The uplink wind option was the main independent variable of interest in this study. Given a three-dimensional wind forecast grid, the uplink option was defined as a combination of:

- the spatial location where the wind data were sampled;

- the specific altitudes where the wind data were sampled; and

- the number of data points, i.e., altitudes, that were sampled.

This data is augmented by the sensed winds at the IM aircraft's current position. The FIM equipment will make use of all wind information provided to build trajectories. Figure 1 shows the RAP model wind forecast at an altitude of $15000 \mathrm{ft}$ in the simulation airspace for two different wind condition. The RAP model wind forecast data was obtained from the National Climate Data Center (NCDC).
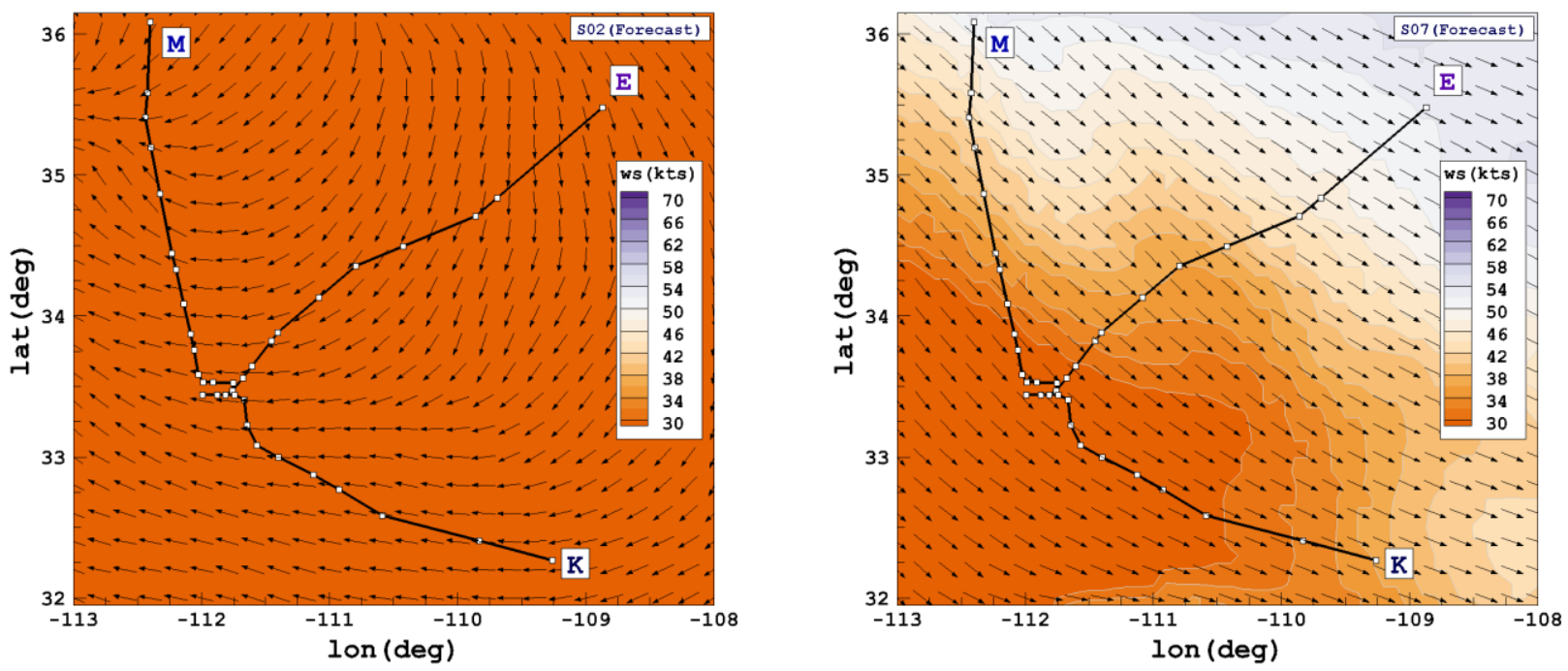

Figure 1: RAP model forecast for Phoenix area at an altitude of $15000 \mathrm{ft}$ for two different days. The arrows indicate the wind direction only. The magnitude of wind speed is given by the color map.

The discrete set of options consisting of the number of data points, the altitudes and the spatial location of those points along with the subset of five selected wind forecast subset options are described below:

\section{Spatial Location}

In all cases, forecast winds at the location and altitude of the achieve-by point are included.

1. Single column at the airport - based at the airport location, the winds at different altitudes are selected and provided to both the IM and the target aircraft.

2. Single column at mid-point on route - at approximately the mid-point of the arrival route, for example, 60 nmi flight distance from the runway, the winds at different altitudes are selected and provided. If the IM and target aircraft are on different arrival routes, their wind forecast data will differ.

3. Along path selection - based on the expected or published vertical profile of each aircraft, a predetermined set of altitudes is selected and provided. If the IM and the target aircraft are on different arrival routes, their wind forecast data will differ.

It is assumed in the FIM Safety, Performance and Interoperability Requirements (SPR) (RTCA, 2011) that wind data will be provided at the expected achieve-by point crossing altitude. For the arrival operations modeled in this study, it is assumed that winds at the end of the runway and at the final approach fix (FAF is used as the achieve-by point), are close enough that no change is needed in option 1 to meet this assumption. Both option 1 and option 3 will be constrained to include a lowest altitude of $3000 \mathrm{ft}$ (cross altitude at the achieve-by point). For option 2, one of the altitude points will be set to $3000 \mathrm{ft}$ at the achieve-by point.

\section{Altitude Selection}

A. A predetermined set - A fixed set of altitudes are used and remain the same for all wind conditions. 
B. Optimized set - The Visvalingam-Whyatt algorithm (Visvalingam and Whyatt 1993) was used to optimally select the altitudes so that the interpolated data based on the limited number of points best matched the actual vertical profile of the headwind component. For the Visvalingam-Whyatt algorithm each point of the polyline describing the full wind forecast as a function of altitude is assigned an effective area. The effective area is the area of the triangle formed by the point and its two neighbors. The point with the smallest effective area is deleted from the list. The method is repeated until the polyline is reduced to the required number of points.

\section{Number of Data Points}

In the near term National Airspace System, it is expected that for FIM in a voice environment, the wind forecast data will consist of three altitudes in addition to the winds at the achieve-by point. Therefore, four altitudes, including the achieve-by point, should be a minimum. A maximum of nine points is assumed. One of those altitude points is set to $3000 \mathrm{ft}$ at or near the FAF. A point at the top of descent at a geographic point determined by the spatial location option is included but not counted against the total number of data points. This ensures that there is wind data bounding all expected altitudes. The draft FIM Minimum Operational Performance Standard (MOPS) being developed by RTCA assumes that the sensed winds at cruise will be used as the highest altitude winds. However, TMX does not currently support the use of sensed winds in the initial forecast data so forecast data was substituted. Since ASTAR blends the sensed winds into the forecast data provided, the cruise altitude forecast point is quickly replaced by the sensed winds at that location.

For all conditions, the full forecast data is extracted from the RAP data either along the flight path or above a fixed point. Then the specified number of points are selected from the extracted wind profile. Figure 2 shows an example of the location and wind speed for the selected altitudes for one of the wind conditions used in this analysis.

\section{Uplink Test Conditions}

The following five test conditions for the uplink wind were selected from the choices described above:

1. $2 \mathrm{~A} 4-3000 \mathrm{ft}$ at the final approach fix, then three fixed altitudes at a geographic point approximately $60 \mathrm{nmi}$ flight distance from the airport plus $35000 \mathrm{ft}$ near the top of descent. The four altitudes are $10000 \mathrm{ft}, 20000$ $\mathrm{ft}, 30000 \mathrm{ft}$, and $35000 \mathrm{ft}$. These altitudes are based on those used for the FIM MOPS wind analysis.

2. $3 \mathrm{~A} 4-3000 \mathrm{ft}$ at the final approach fix, then four altitudes along the expected flight path ending with altitude at the top of descent. The four altitudes are $8000 \mathrm{ft}, 16000 \mathrm{ft}, 24000 \mathrm{ft}$, and the altitude at the top of the descent. These are approximately evenly spaced but with a preference for lower altitudes as those tend to have the largest impacts.

3. $3 \mathrm{~A} 9-3000 \mathrm{ft}$ at the final approach fix, then nine altitudes along the expected flight path ending with 35000 $\mathrm{ft}$ near the top of descent. The nine altitudes are $6000 \mathrm{ft}, 9000 \mathrm{ft}, 12000 \mathrm{ft}, 15000 \mathrm{ft}, 18000 \mathrm{ft}, 22000 \mathrm{ft}, 26000$ $\mathrm{ft}, 30000 \mathrm{ft}$, and the altitude at the top of the descent. These are spaced $3000 \mathrm{ft}$ apart below $18000 \mathrm{ft}$ and 4000 $\mathrm{ft}$ apart above $18000 \mathrm{ft}$ except for the altitude point at the top of descent.

4. 3B4 - Five (four plus the one at top of descent) optimized altitudes along the route anchored by $3000 \mathrm{ft}$ and the altitude at the top of descent.

5. $3 \mathrm{~B} 9$ - Ten (nine plus the one at top of descent) optimized altitudes along the route anchored by $3000 \mathrm{ft}$ and the altitude at the top of descent.

All altitudes are above mean sea level. The code for each condition is comprised of three main parts. The first element is a number that indicates whether the winds were sampled at a single column (denoted by the number 2), or if they were sampled along the aircraft's intended trajectory (denoted by the number 3 ). The second element in the code indicates whether a standard set of locations and altitudes were used (denoted by the letter A), or if an optimization routine was used to select the optimal set of altitudes and locations (denoted by the letter B). The last element in the code indicates the number of sampling points. 

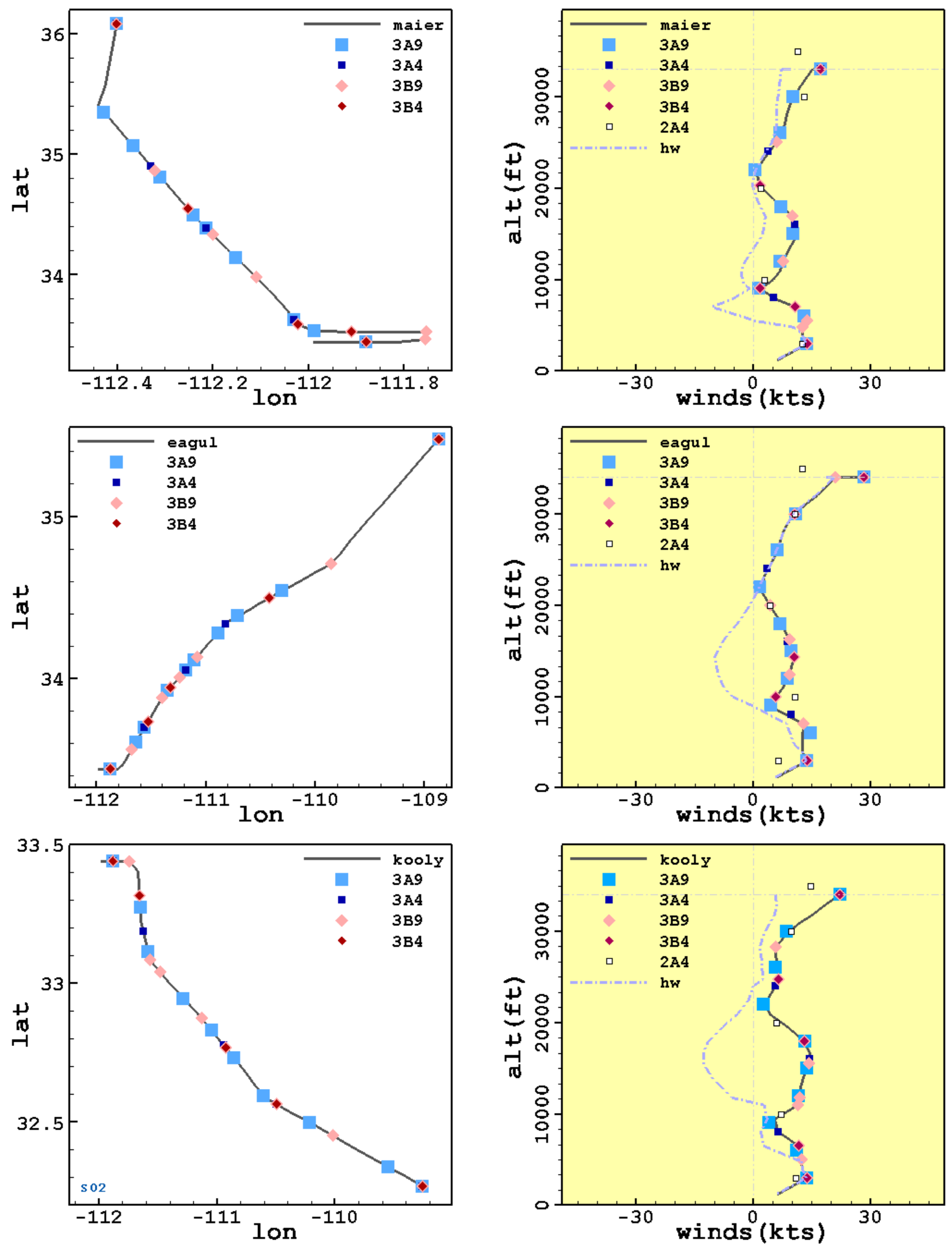

Figure 2: Wind uplink options for wind condition 2. From top to bottom are the MAIER5, EAGUL5, and KOOLY4 routes. Points in the plots represent the uplinked data. Solid lines in the left column show the routes and the points mark the spatial location of the uplink data. Solid lines in the right column denote the winds along the route and dashed lines are the headwind component. 


\section{Test Variables}

The variable of interest for this study is the uplink option as enumerated in section II.B.4.

Each data run consisted of one non-IM aircraft (the lead aircraft) followed by five IM aircraft. It is assumed that data from consecutive pairs of aircraft in a string are weakly correlated and therefore can be analyzed as independent samples. To represent the expected performance across an extended period of time, an average over many conditions was required. The following conditions were included in this study:

- Wind conditions (10 options sampled equally, see Table 1)

- Lead speed profile (5 options sampled equally, Swieringa et al. 2014)

- Route assignment (uniform with no repetition; Section III A)

- Aircraft type (uniform from limited set; see Table 2)

- Initial delay (Gaussian, mean $=30 \mathrm{sec} ; \sigma=20 \mathrm{sec}$ ). The initial delay is the difference between the actual initiation time and the time that would be expected if the aircraft would fly the published speed profile and arrive at the scheduled time of arrival (STA).

- Aircraft weight (uniform; see Table 2)

The wind conditions (pairs of truth and forecast wind grids) were provided by MIT's Lincoln Lab (Troxel 2014). The wind data is based on MIT's climatological analysis of one year's RAP data covering the entire Continental United States (Table 1). The forecast error for each scenario was assigned by MIT-LL based on the difference between the truth and forecasted headwinds along each route.

Table 1: Weather Scenarios.

\begin{tabular}{|l|l|l|l|l|l|}
\hline Scenario & \multicolumn{2}{|c}{ Truth Data } & \multicolumn{4}{|c|}{ Forecast Data } & \multicolumn{1}{|c|}{ Forecast } \\
& Date & Time & Date & Time & Lror \\
\hline $\mathbf{1}$ & $2014 / 05 / 17$ & $18: 00$ & $2014 / 05 / 17$ & $16: 00$ & Low \\
\hline $\mathbf{2}$ & $2014 / 07 / 18$ & $6: 00$ & $2014 / 07 / 18$ & $4: 00$ & Low \\
\hline $\mathbf{3}$ & $2014 / 07 / 31$ & $6: 00$ & $2014 / 07 / 31$ & $4: 00$ & Low \\
\hline $\mathbf{4}$ & $2013 / 09 / 11$ & $12: 00$ & $2013 / 09 / 11$ & $10: 00$ & Moderate \\
\hline $\mathbf{5}$ & $2013 / 11 / 08$ & $12: 00$ & $2013 / 11 / 08$ & $10: 00$ & Moderate \\
\hline $\mathbf{6}$ & $2014 / 02 / 02$ & $6: 00$ & $2014 / 02 / 02$ & $4: 00$ & Moderate \\
\hline $\mathbf{7}$ & $2014 / 01 / 04$ & $18: 00$ & $2014 / 01 / 04$ & $16: 00$ & High \\
\hline $\mathbf{8}$ & $2014 / 03 / 05$ & $12: 00$ & $2014 / 03 / 05$ & $10: 00$ & High \\
\hline $\mathbf{9}$ & $2014 / 07 / 18$ & $12: 00$ & $2014 / 07 / 18$ & $10: 00$ & High \\
\hline $\mathbf{1 0}$ & $2013 / 11 / 23$ & $6: 00$ & $2013 / 11 / 23$ & $4: 00$ & Extreme \\
\hline
\end{tabular}

Five representative speed profiles for the lead aircraft were used to represent expected behavior from a controller using future automation. Aircraft type was selected from the available BADA models (see Table 2). Aircraft weight was also selected from BADA reference mass plus 0-50\% max payload. This is a rough estimate of acceptable landing weights as BADA does not provide landing weight information. 
Table 2: Aircraft types and weight range.

\begin{tabular}{|l|lll|l|}
\hline Aircraft Type & $\begin{array}{l}\text { Reference } \\
\text { Mass (t) }\end{array}$ & $\begin{array}{l}\text { Max } \\
\text { Payload }\end{array}$ & minimum & maximum \\
\hline B737-700 & 60 & 16.9 & 60 & 68.45 \\
\hline B777-300 & 238 & 64.9 & 238 & 270.45 \\
\hline A320 & 64 & 21.5 & 64 & 74.75 \\
\hline A319 & 60 & 17 & 60 & 68.5 \\
\hline B757-200 & 95.3 & 21.4 & 95.3 & 106 \\
\hline A306 & 280 & 78 & 280 & 319 \\
\hline
\end{tabular}

Based on a power analysis, a minimum of 200 data points per test condition was needed to detect at least a 1 second difference in the mean delivery error. For each test condition, the full set of ten wind conditions and five lead speed profiles were used. Strings of five IM pairs resulted in a total of 250 data samples per test condition.

\section{Simulations}

As discussed below, the results were not as definitive as expected. Therefore, additional runs were performed in an attempt to eliminate sources of uncertainty that could have masked the expected effect. This paper focuses on Run 1 , the original design, with comments in the analysis on the effects of the modifications for runs 2-4. Full presentation of runs 2-4 will be forthcoming in a future report.

The four sets of simulations were:

- Run 1: Baseline simulation as described in the previous sections. This has the full range of variability and the target aircraft following delay trajectories that are unknown to the IM Aircraft (expected condition for initial IM deployment).

- Run 2: Nominal speed profiles were used for the target aircraft and all other parameters were kept the same as in Run 1 . This has the full range of variability except that the target aircraft is following the speed profile that is given to the IM aircraft. This condition was designed to emulate an Advanced IM environment where the specific speed profile of the target aircraft can be communicated to the IM Aircraft.

- Run 3: Nominal speed profiles were used for the target aircraft. No wind forecast error was assumed (all other parameters were same as in Run 1). This removes the forecast winds as a source of uncertainty. The results focus on the effects of having a discrete forecast sent to the IM Aircraft.

- Run 4: Nominal speed profiles were used for the target aircraft. No wind forecast error was assumed. A total of 60 wind conditions were used which increased the total number of scenarios from 250 to 1500 (all other parameters were same as in Run 1). This extends Run 3 to include a wider range of wind conditions.

The dates for the additional 60 wind conditions were provided by MITRE and match the selected wind conditions used in the FIM MOPS analysis. These 60 wind conditions were in addition to the wind scenarios provided by MIT. The Run 4 condition was added to determine if some of the unexpected behavior seen was a result of using winds from a small sampling of days.

\section{Data Analysis}

Analyses of the following key metrics is presented in this section:

1. Delivery error - the difference between the achieved spacing and the assigned spacing goal at the achieveby-point. Negative numbers indicate that the achieved spacing was less than the assigned spacing goal.

2. RMS of IM speed deviation - The root-mean-square (RMS) value of the difference between the IM Speed and the profile speed. This provides a measure of how much control the algorithm needed to apply to achieve the assigned spacing goal. 
3. Number of speed commands - a count of the number of times the IM speed changes as commanded by the ASTAR algorithm.

A total of 1250 data points were collected with 250 data points for each of the five uplink conditions. Distributions of data are shown below as a combined box-and-whisker plot with a mean-standard deviation overlay. The mean and standard deviation are shown by a green rectangle extended plus and minus the standard deviation away from the central green line representing the mean of the distribution. The box portion shows the median (red vertical line) and the interquartile distance in both directions. The interquartile distances represent the 25-50 percentile and 50-75 percentile of the data and are not necessarily equal in size. The notch in the box is an estimate of significant differences. If the median of one distribution falls outside the notch on another, then the difference between the two distributions is statistically significant. On both sides of the box the whiskers extend 1.5 times the interquartile distance on that side. Any data points falling outside the whiskers are shown individually as a red cross.

\section{A. Delivery Accuracy and Precision}

The delivery accuracy and precision are measures of how precisely the IM algorithm is able to achieve the desired spacing goal. Previous work has shown that the delivery error roughly follows a Gaussian distribution, at least for the central peak. Therefore, summary statistics are shown for the delivery error distribution as mean plus standard deviation. However, since it is the tails of the distribution that affects safety and throughput, the central $95 \%$ of the data, symmetric around the median, is also shown. The summary statistics of delivery accuracy for Run 1 are shown in Table 3. Uplink option 3B9 had the smallest mean in delivery accuracy while option 3A9 had the smallest standard deviation. Figure 3 shows the box-and-whisker plot for Run 1 for each of the uplink options.

Table 3: Run 1 - Delivery Error at Final Approach Fix statistics.

\begin{tabular}{|c|c|c|c|c|}
\hline Option & \multicolumn{1}{|c|}{ mean(s) } & \multicolumn{1}{c|}{$\boldsymbol{\sigma}(\mathbf{s})$} & median(s) & $\mathbf{9 5 \% ( s )}$ \\
\hline $\mathbf{2 A 4}$ & 1.809 & 2.719 & 1.800 & 11.225 \\
\hline $\mathbf{3 A 4}$ & 1.921 & 2.798 & 1.500 & 11.375 \\
\hline $\mathbf{3 B 4}$ & 1.606 & 2.507 & 1.300 & 10.300 \\
\hline 3A9 & 1.973 & 2.368 & 1.800 & 8.350 \\
\hline 3B9 & 1.562 & 2.624 & 1.500 & 8.850 \\
\hline
\end{tabular}

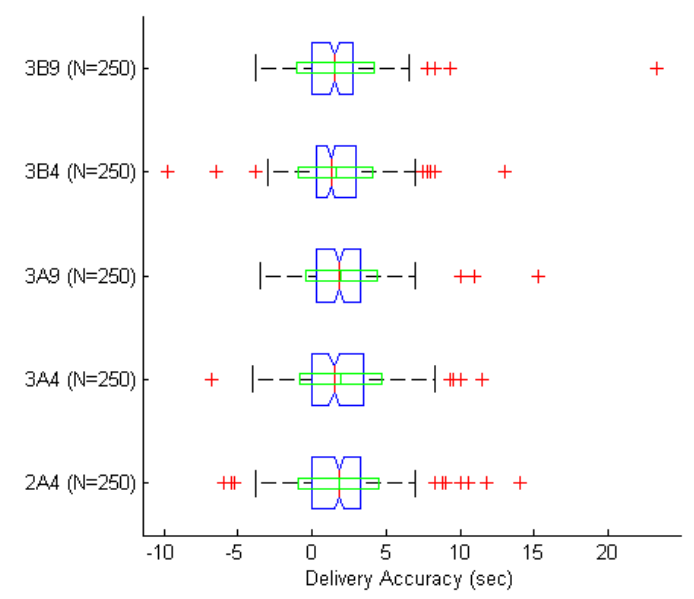

Figure 3: Box-and-whisker plot for delivery error across the five uplink options for Run 1.

For all four runs (Section II D), ANOVA tests were performed to identify any statistically significant differences in the mean delivery error $(\mathrm{p}<0.05)$. The tests identified statistically significant differences between options $3 \mathrm{~A} 4$ and both 3A9 and 3B9 in Run 3. In Run 4, options 3B9 and 3A4 were determined to have statistically significant differences. No statistically significant differences were found in delivery error as a function of uplink option in Runs 
1 and 2. Statistically significant differences were also found in delivery error as a function of uplink points (four vs. nine) in Runs 3 and 4.

For Run 1 and 2, those where the wind model had forecast errors, only the options with 9 altitudes, 3A9 and 3B9, met the performance goal of having $95 \%$ of aircraft delivered within a \pm 10 second bound. When the forecast error was removed from the wind model, Runs 3 and 4, all uplink options met the 10 second, 95\% goal. However, options 3A9 and 3B9 continued to have small $95 \%$ bounds, indicating that the delivery accuracy distributions for the 3A9 and 3B9 wind uplink options had fewer outliers in the tails of their distributions than the other wind uplink options. One possible explanation for this is that the large number of forecast points enabled a more accurate prediction of the IM aircraft's ETA and the target aircraft's ETA, and provided a more accurate wind forecast close to the achieve-by point. In some cases, this combination could have prevented large spacing errors from occurring when the IM aircraft is close to the achieve-by point.

\section{B. Root Mean Square of Speed Control}

The RMS of the speed control throughout the arrival was examined to determine if particular wind forecast uplink options required less speed control than others. The RMS of the speed control is sensitive to the magnitude of the initial spacing error; however, the initial spacing errors for each wind forecast method were sampled from the same distribution. Thus, an increase in the RMS of the speed control indicates less optimal performance.

Statistics summaries for Run 1 are given in Table 4. Figure 4 shows the box-and-whisker plot for Run 1 for each of the uplink options.

Table 4: Run 1 - RMS of difference between IM Speed and profile speed.

\begin{tabular}{|l|l|l|l|l|}
\hline Option & mean(kts) & $\boldsymbol{\sigma}(\mathbf{k t s})$ & $\operatorname{median}(\mathbf{k t s})$ & $\mathbf{9 5 \%}(\mathbf{k t s})$ \\
\hline $\mathbf{2 A 4}$ & 7.702 & 3.508 & 7.184 & 14.066 \\
\hline $\mathbf{3 A 4}$ & 9.393 & 4.099 & 8.744 & 16.119 \\
\hline $\mathbf{3 B 4}$ & 8.190 & 3.097 & 7.610 & 11.824 \\
\hline $\mathbf{3 A 9}$ & 8.583 & 3.326 & 8.060 & 12.829 \\
\hline $\mathbf{3 B 9}$ & 7.965 & 3.116 & 7.771 & 11.781 \\
\hline
\end{tabular}

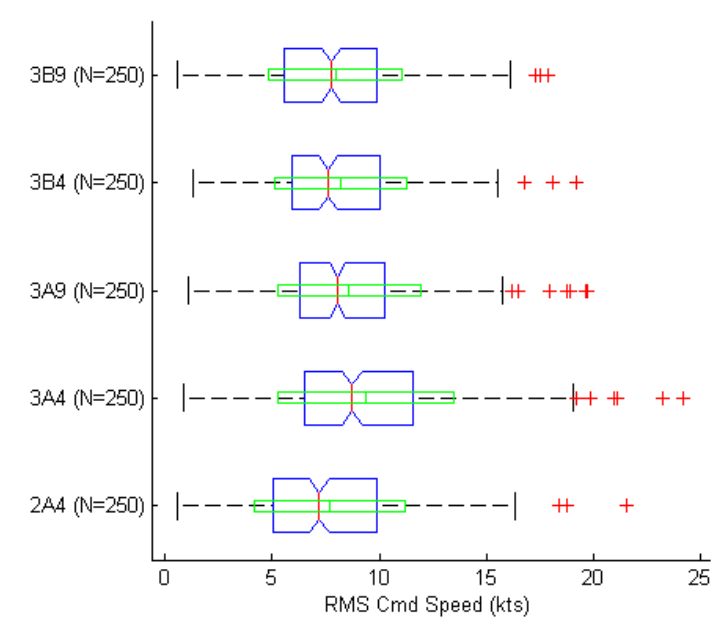

Figure 4: Box-and-whisker plot for RMS speed difference across the five uplink options for Run 1.

ANOVA tests were performed to look for differences between the five uplink options. Statistically significant differences between conditions were found in all four runs (Section II D). In Runs 1 and 2 the differences between the means of groups $3 \mathrm{~A} 4$ and $3 \mathrm{~A} 9$ were statistically significant from 2A4 and the differences between the means of 
both 3B4 and 3B9 wind uplink options were statistically significantly lower than the 3A4 wind uplink option. In Runs 3 and 4 the differences between the means of groups 2A4, 3B4 and 3B9 were statistically significant from 3A4.

The results do not show a large difference in the average amount of speed control; however, it is interesting to note that the wind uplink options that used an optimization routine to select the locations and altitudes where the winds were sampled (condition 3B4 and 3B9) had a lower $95^{\text {th }}$ percentile value than the other wind uplink options. Additionally, examining the subset of wind uplink options that did not use the optimization routine indicates that the wind uplink option with nine points (3A9) required less speed control than the non-optimized wind uplink options with fewer points. One explanation for this data is that a majority of the wind fields have very little variability, allowing them to be accurately sampled by any of the uplink options that were investigated. However, more complex wind patterns require either a greater number of points to sample them accurately or for the locations and sample points to be chosen to maximize the accuracy of the forecast. Thus, using a high number or sampling points or optimizing will provide more consistent performance across a wider range of wind conditions; particularly those with more complex structure.

\section{Number of Speed Commands}

The number of speed changes commanded by the FIM Equipment is used as a proxy for the workload on the flight crew to perform the IM operation after the operation has begun. The statistics summaries for total number of speed changes per flight are given in Table 5 for Run 1. Figure 5 shows the box-and-whisker plot for Run 1 for each of the uplink options.

Table 5: Run 1 - Total number of speed changes per flight.

\begin{tabular}{|l|l|l|l|l|}
\hline Option & mean & \multicolumn{1}{c|}{$\boldsymbol{\sigma}(\mathbf{k t s})$} & \multicolumn{1}{c|}{ median } & $\mathbf{9 5 \%}$ \\
\hline $\mathbf{2 A 4}$ & 9.98 & 1.67 & 10 & 6 \\
\hline $\mathbf{3 A 4}$ & 10.82 & 2.11 & 11 & 8 \\
\hline 3B4 & 10.61 & 2.02 & 11 & 8 \\
\hline 3A9 & 10.49 & 1.88 & 10 & 7 \\
\hline 3B9 & 10.03 & 1.77 & 10 & 7 \\
\hline
\end{tabular}

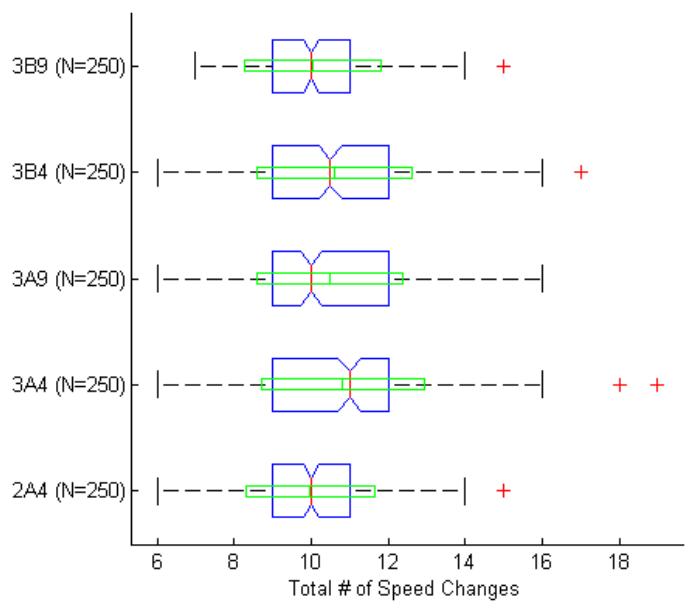

Figure 5: Box-and-whisker plot for total number of speed changes across the five uplink options for Run 1.

ANOVA tests revealed several statistically significant differences in the mean number of speed changes over the whole operation. Option 2A4 was statistically different from options 3A4, 3A9, and 3B4. Option 3B9 was statistically different from option 3A4 and 3B4. The test for differences in the number of speed changes has strong statistical 
power and is therefore apt to detect differences that are not operationally relevant. The mean number of speed changes never varied by more than one per arrival operation.

\section{Summary}

A fast-time simulation was conducted to examine five wind forecast uplink options to determine the effects of the uplink options on three key performance metrics. The uplink options were identified based on location, number of points and the altitudes sampled. Performance was compared for three metrics: the delivery accuracy, number of speed commands, and the total amount of speed control used throughout an arrival.

It was expected that the 2A4 uplink option would have worse performance, since it uses the fewest number of altitudes, the altitudes are pre-selected and likely miss the actual structure of the wind profile and all winds are sampled at a single geographic point instead of along the flight profile. Option 3A4 was expected to be a slight improvement as the forecast data would be sampled along the expected flight trajectory. Both the 3A9 and 3B4 options were expected to further improve performance by providing better representations of the wind profile. Option 3A9 by including samples at five additional altitudes and option 3B4 by optimally selecting the altitudes to sample. Finally, option 3B9 combined all of these improvements and was thus expected to provide the best performance. This was not the case. For most metrics and runs there was no statistically significant differences between the uplink conditions.

A one-way ANOVA test combined with t-tests showed no significant differences for the uplink option in delivery accuracy. This was unexpected as increasing the number of altitudes sampled, using a set of altitudes optimized to best represent the actual profile shape and sampling at points along the expected vertical path were all assumed to improve the overall performance. When removing the uncertainty added by forecast errors in the forecast model, a small difference was detected between the 3A4 and 3B9 uplink options across the full 70 wind conditions (Run 4). This difference was only 0.3 seconds in the mean and median so the operational impact of the difference would be small.

Looking at the $95 \%$ bounds on delivery error does reveal some differences between the uplink options. The 3A9 and 3B9 options consistently show a smaller $95 \%$ bound suggesting that the greater number of forecast data reduces the number of outliers. While the operational benefits of small changes in the mean delivery may be small, a reduction in the $95 \%$ bounds of $2-3$ seconds would be operationally significant. A similar trend can be seen in the other metrics, although not as pronounced.

The means of uplink options 3A9 and 3B4 were significantly different in RMS commanded speeds. The RMS metric is a relatively new attempt to assess the amount of control needed to meet the final delivery. While the delivery error metric measures the end state, the RMS of the differences in speeds attempts to capture the dynamics of the entire operation into one system-level metric. Because this is a relatively new metric of consideration, it is not known what the operationally relevant differences are. For this metric in particular, the 3A4 uplink option was consistently larger than the other options.

Significant differences were observed between different uplink options in the total number of speed change commands. However, the statistical tests for this metric, since is a discrete value instead of continuous, is particularly powerful and likely to identify very slight differences. The differences in the mean and median were never more than one speed change as was the $95 \%$ range.

Table 6: Statistically significant differences based on location and points.

\begin{tabular}{|c|l|l|l|l|}
\hline \multirow{2}{*}{ Run } & \multicolumn{2}{c}{$\begin{array}{c}\text { Location } \\
\text { along-route/fixed }\end{array}$} \\
\cline { 2 - 5 } & delivAcc (s) & RMS Spd Cmd (kts) & delivAcc (s) & RMS Spd Cmd (kts) \\
\hline $\mathbf{1}$ & $1.76 / 1.81$ & $\mathbf{8 . 5 3 / 7 . 7 0}$ & $1.77 / 1.76$ & $8.42 / 8.27$ \\
\hline $\mathbf{2}$ & $1.78 / 1.83$ & $\mathbf{8 . 5 2 / 7 . 6 9}$ & $1.80 / 1.77$ & $8.41 / 8.27$ \\
\hline $\mathbf{3}$ & $1.72 / 1.66$ & $\mathbf{8 . 0 0 / 7 . 3 7}$ & $\mathbf{1 . 8 2 / 1 . 5 5}$ & $\mathbf{8 . 1 5 / 7 . 4 7}$ \\
\hline $\mathbf{4}$ & $1.77 / 1.81$ & $\mathbf{7 . 6 2 / 7 . 2 8}$ & $\mathbf{1 . 8 5 / 1 . 6 8}$ & $\mathbf{7 . 7 2 / 7 . 2 9}$ \\
\hline
\end{tabular}


There are at least two possible explanation for the limited differentiation between the uplink conditions. First, the smallest samplings, four altitudes, may provide all of the information content needed to reduce the effects of the discrete forecast data to below other sources of spacing uncertainty. However, simulations done in support of the FIM MOPS, using a different FIM algorithm, showed improvement when adding more altitudes to the discrete forecast data. A similar trend can be seen when collapsing the data across location and altitude selection and just focusing on the number of altitudes provided (see Table 6). In the table, those differences that are statistically significant are shown in red text. Also, ASTAR constantly blends the IM Aircraft's sensed winds into the internal wind model so ASTAR is partially correcting the forecast data to match the sensed data. While the effect of this blending is limited in range, it partially offset the effects of wind forecast errors.

The second possible explanation is that the winds in the Phoenix area appear to be rather smooth and similar across the routes studied. As can be seen in Figure 2, there are only small variations along the routes, reducing the difference between sampling along the flight profile or at a single geographic point, and the vertical profile has little structure, reducing the impact of the number of altitudes selected and the specific altitudes. In fact, as Figure 6 shows for wind condition 7, the vertical profiles along all three arrival routes are nearly straight lines so only two altitudes would be needed to describe the winds. Extending this study to a location such as Denver which is expected to have at least greater spatial variability may show greater differences between the uplink options. Simulations using idealized wind profiles with variability along the three routes used in the current study are also planned in order to quantify the differences between different uplink options.
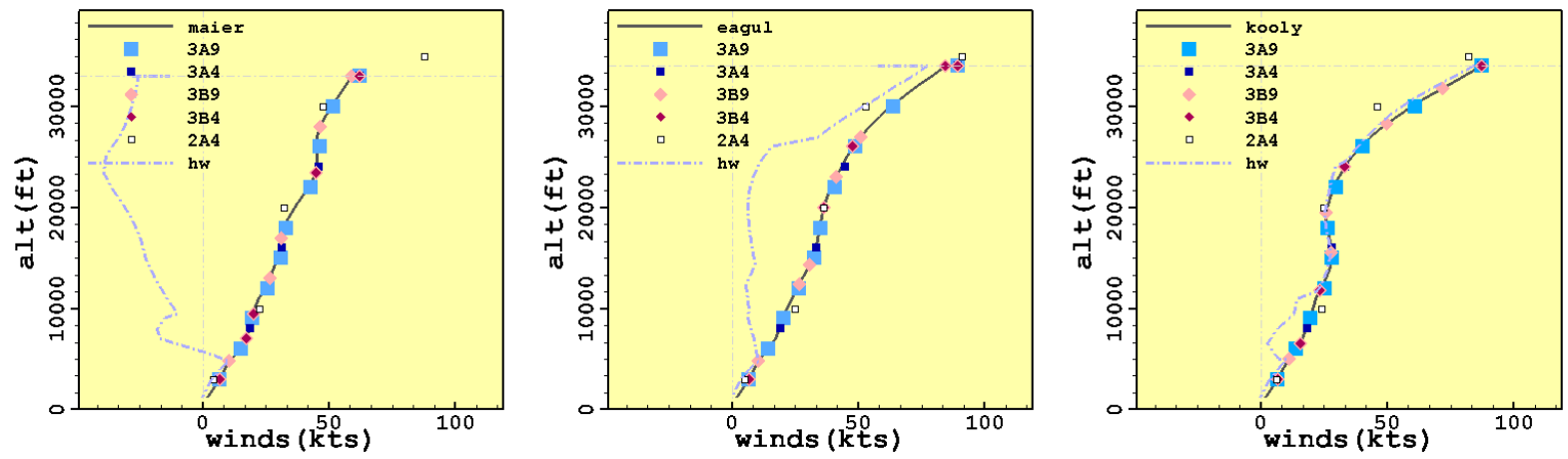

Figure 6: Wind uplink options for wind condition 7. From left to right are the MAIER5, EAGUL5, and KOOLY4 routes. Points in the plots represent the uplinked data. Solid lines denote the winds along the route and dashed lines are the headwind component.

Additional runs were performed in an attempt to eliminate sources of uncertainty such as wind forecast error that could have masked the expected effect of uplink information. This paper focused on Run 1, the original design, with brief discussion on the effects of the modifications for runs 2-4 (Section II.D). Full presentation of runs 2-4 will be forthcoming in a future report.

\section{Acknowledgement}

Many thanks to Seth Troxel (MIT-Lincoln Lab) for identifying the weather scenarios described in Table 1.

\section{References}

Abbott, TS, “An Overview of a Trajectory-Based Solution for En Route and Terminal Area Self-Spacing: Fifth Edition", National Aeronautics and Space Administration, 2015, NASA/CR-2015-218680.

Barmore, BE, TS Abbott, and K Krishnamurthy, "Airborne-Managed Spacing in Multiple Arrival Streams," International Congress of Aeronautical Sciences, 2004.

Barmore, BE, RS Bone, and WJ Penhallegon, "Flight-deck merging and spacing operations," Air Traffic Control Quarterly, Vol. 17, no. 1, 2009, pp. 5-37.

Bussink, FJL, Hoekstra, J, Heesbeen, W, “Traffic Manager: A Flexible Desktop Simulation Tool Enabling Future ATM Research,” 24th Digital Avionics Systems Conference, October 2005. 
Hershberger, J, J Snoeyink, "Speeding Up the Douglas-Peucker Line-Simplification Algorithm", Proceedings of the $5^{\text {th }}$ International Symposium on Spatial Data Handling, 1992, pp. 134-143.

Murdoch, JL, BE Barmore, BT Baxley, TS Abbott, and WR Capron, "Evaluation of an airborne spacing concept to support continuous descent arrival operations," in Proceedings of the ATM R\&D Seminar, 2009.

Nuic, A, "User Manual for the Base of Aircraft Data (BADA) - Revision 3.5," Eurocontrol Experimental Centre, Bretigny, France, http://www.eurocontrol.int/eec/public/standard_page/ACE_bada.html. 2003.

Shi, W, C Cheung, "Performance Evaluation of Line Simplification Algorithms for Vector Generalization", The Cartographic Journal, Vol. 43, 2006, pp. 27-44.

RTCA Inc., "Safety, Performance and Interoperability Requirements Document for Airborne Spacing - Flight Deck Interval Management (ASPA-FIM),” ED-195/DO328 Rev A, Draft V1.0, 2011, pp. 583.

Swieringa, KA, SR Wilson, R Shay, “An Evaluation of a Flight Deck Interval Management Algorithm including Delayed Target Trajectories,", AIAA Paper 2014-3148.

Troxel, S., "Wind Scenarios for NASA ATC IM Winds Study", Private Communication. August 2014.

Visvalingam, M, JD Whyatt, 'Line generalization by repeated elimination of points', The Cartographic Journal, Vol. 30, 1993, pp. $46-51$.

Weitz, LA, R Katkin, P Moertl, WJ Penhallegon, JB Hammer, RS Bone, and T Peterson, "Considerations for interval management operations in a mixed-equipage environment," in Proceedings of the AIAA Aviation, Technology, Integration, and Operations Conference, 2012. AIAA Paper 2012-5616. 